\title{
Sistema médico tradicional- ancestral en el territorio de los Pastos Resguardo Indígena de Túquerres-Narino, Colombia
}

\author{
Sandra Patricia Portilla Rodríguez ${ }^{1}$ \\ Orlando Madroñero ${ }^{2}$ \\ Pablo Emilio Getial ${ }^{3}$
}

\section{Resumen}

Se ha analizado el sistema médico tradicional en el territorio de los Pastos, Resguardo Indígena de Túquerres, Nariño, Colombia. Para los indígenas, la salud es producto de la relación armónica entre naturaleza, seres humanos y el mundo espiritual, al romperse uno de estos tres nexos, el cuerpo se enferma afectando tanto al individuo como a la familia.

El enfoque fue hermenéutico en la comprensión de las prácticas de los comunitarios relacionadas con la medicina tradicional. La unidad de estudio fue la totalidad de médicos tradicionales (19), entre los cuales hay hierbateros, espirituales, yageceros y parteras. Los resultados han reflejado que la práctica de la medicina tradicional, que en el caso del Estado, algunos sectores de la Iglesia e Instituciones prestadoras de salud no aceptan esta práctica ancestral, persiguiendo al médico tradicional acusándolo de hechicero, embustero y mentiroso.

Existen dos categorías de médicos tradicionales: los espirituales y los del cuerpo. Entre las más enfermedades más frecuentes del cuerpo está la gastritis, la úlcera, apendicitis, mal de vesícula, cáncer, fracturas y luxaciones. Entre las espirituales: el espanto, el mal viento, el miado del cueche, el maleficio y enfermedades que denominan "del alma", como la envidia, la soledad, la tristeza, el egoísmo.

El tratamiento va acompañado con un ritual. Para las espirituales se purifica a la persona y el entorno con sahumerio, se invoca a Dios y naturaleza, sol, agua, y los espíritus mayores. La percepción comunal: consideran que la medicina tradicional es una alternativa para la salud del cuerpo y el espíritu a través de plantas.

Palabras Clave: medicina tradicional; espiritualidad; cosmovisión; identidad.

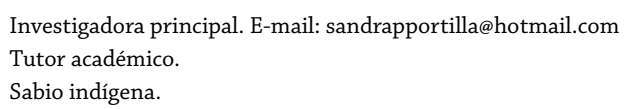




\section{Summary}

We have analyzed the traditional medical system in Los Pastos territory, Indigenous Reservation of Tuquerres, Nariño, Colombia. For indigenous people, health is a product of the harmonious relationship between nature, human beings and the spiritual world, so when one of these three links breaks, the body becomes sick, affecting both the individual and the family.

We use a hermeneutical approach to understand the community practices related to traditional medicine. The unit of study was the entire traditional doctors (19), among whom we can mention herbalist's (bush doctors), spiritualist, medicine man (yageceros) and midwives. The results have reflected that the practice of traditional medicine, in the case of the State, some sectors of the Church and institutions that provides health care do not accept this ancestral practice, but rather they chase the traditional doctor accusing him as a witch doctor, a deceitful and a liar.

There are two categories of traditional doctors: those that address the spiritual part and those that attends the body. Among the most common diseases of the body we can find gastritis, ulcer, appendicitis, vesicle disease, cancer, fractures and dislocations. Between the spiritual ones: the fright, the bad wind, the piss of the cueche, the curse and those known as the "diseases of the soul", like the envy, the loneliness, the sadness and the selfishness.

The treatment is accompanied with a ritual. For the spiritual ones the person and the environment are purified with incense, while they invokes God and nature, sun, water, as well as the greater spirits. On the other side, the communal perception considers that the traditional medicine is an alternative for the health of the body and the spirit through plants.

Keywords: traditional medicine; spirituality; worldview, identity.

\section{Introducción}

Para los indígenas la salud es entendida como el producto de la relación armónica o equilibrio existente entre la naturaleza, los seres humanos y el mundo espiritual, al romperse uno de estos tres nexos, el cuerpo se enferma afectando tanto al individuo como a la familia. En este aspecto la enfermedad y la salud no sólo son definidas en un sentido individual sino en un sentido social; para contrarrestarlas es necesario restaurar la armonía perdida, buscando el favor de los espíritus, de la naturaleza y recurriendo a las propiedades de las plantas.

Dentro del resguardo indígena de Túquerres, Nariño, Colombia, las condiciones de marginalidad y pobreza afectan la salud vulnerando los derechos propios, armonía 
y bienestar de sus pobladores. La desatención estatal y la falta de eficiencia, calidad y oportunidad en la prestación de los servicios de salud agravan la situación. Es por eso que los índices de mortalidad y morbilidad en el resguardo son más altos que los promedios regionales y nacionales.

En consecuencia, el sistema propio de salud debe estar construido desde la cosmovisión indígena de cada pueblo en defensa de su cultura para ser gobernado por los indígenas de acuerdo al derecho propio, jurisdicción especial, Constitución Política nacional, sus derechos normativos y el derecho internacional de los pueblos originarios. En el marco de un relacionamiento crítico, respetuoso y equitativo con la medicina tradicional y occidental, este hecho demuestra la necesidad y pertinencia de ejecutar el presente trabajo de investigación.

La investigación reporta la utilidad práctica y teórica, del estado actual de la medicina tradicional en la comunidad objeto de estudio, acción que permitirá el reconocimiento de esta práctica sociocultural de la comunidad indígena, a la vez que contribuirá con la preservación de este patrimonio cultural milenario.

\section{Revisión de literatura}

\section{La medicina tradicional}

Una característica básica de la medicina tradicional es su fuerte y necesaria vinculación con la cultura del ser, tanto individual como social-grupal. La tradición se convierte en la depositaria y transmisora del saber acumulado y legado a través de generaciones, saber que constituye uno de los pilares básicos en la definición de identidad de los grupos originarios de América, Asia. (Zubiria, R. 1999).

Las tradiciones terapéuticas no occidentales no son sólo elementos que ayudan a perpetuar la cultura y a mantener la cohesión e identidad de los grupos, también curan, es decir, que tienen, como tales, fines y objetivos concretos y específicos, el principal: prevenir la enfermedad y ayudar al restablecimiento de la salud de sus gentes (Bernal, 1991).

\section{Cosmovisión del resguardo de Tuquerres}

Según el Plan de vida del resguardo de Tuquerres (2009); después del territorio, la shagra o cultivo se constituye en el segundo elemento alrededor del cual gira la vida del pueblo indígena, de acuerdo con la Ley de origen, el derecho mayor, el derecho natural y nuestros usos y costumbres, se constituye en el epicentro para el desarrollo de procesos como: economía, educación, cultura, producción, salud, autonomía, entorno natural, espiritualidad, espacialidad, dinamismo que crece y cambia con el pasar del tiempo y el caminar en el espacio. 
La parcela que produce lo necesario para el sustento de la familia y el trueque, permite que en su entorno se forjen los procesos de fortalecimiento de los principios y valores ancestrales. El fogón se convierte entonces en la escuela, los padres en los primeros profesores quienes enseñan, planean, evalúan, aplican justicia y dan amor a sus guaguas, de aquí resulta: la minga como la unión del hogar, la familia, la comunidad símbolo de reunión y conversatorio para labor de beneficio propio o colectivo.

\section{La espiritualidad}

Manifestada en la cosmovisión, el churo y el sol pachianti; el churo es el pensamiento propio, el sol es el dios material de la mitología como son el duende, el chispas, el guangas, el tesmóforo, el Iboag, la vieja, la viuda, el guagua auca, el descabezado, el cuco, el carro de la otra vida, el cuece, la malahora, el mal viento.

El Allpapi es el espacio histórico y espiritual que constituye la casa grande del pueblo indígena de Tuquerreños y Pastos, donde viven agrupados en parcialidades, reproduciendo la identidad cultural, tienen formas de organización propia, las mismas que se encargan de reglamentar la vida colectiva con base a la administración de la justicia propia.

Según Zuluaga (1999), los sitios sagrados cumplen un papel importante en la constitución y el ordenamiento territorial, ya que son estos los que determinaran que este territorio nos pertenece. En los sitios sagrados, se concretan las normas que ancestralmente han definido el uso y manejo del territorio de una cuenca hidrográfica y de las relaciones e interconexiones existentes entre todas las demás cuencas, básicas para materializar la integralidad del resguardo Indígena de Túquerres, concepto fundamental de la cosmovisión indígena y del ordenamiento territorial.

La comunidad y el individuo deben procurar mantener el equilibrio y armonía con la naturaleza y con los demás seres humanos, para lo cual cuentan con médicos tradicionales, curanderos y ellos son los encargados de negociar con los dueños espirituales de los animales, las plantas y de todo lo que existe, para tomar de la naturaleza lo que la comunidad necesita para no romper dicho equilibrio.

Actitudes míticas, creencias, ideologías y la misma identidad de quienes pertenecen a la comunidad contribuirán a perpetuar a lo largo del tiempo un resguardo indígena arraigado a formas de actuar en la que la mujer aún debe conservar su estatus social, el cual lo da el matrimonio y la convivencia formal con un solo compañero sexual.

\section{Metodología}

Esta investigación tuvo el enfoque etnográfico y hermenéutico. Según Briones (1998), estas investigaciones tienen por objeto interpretar o evidenciar cómo se relacionan 
los seres humanos en el mundo social que han creado, se basa en que la vida social que se crea y se mantiene a través de interacciones simbólicas y patrones de conducta que hay que interpretar.

Por tratarse de una investigación cualitativa, este estudio tomó como universo a toda la comunidad de los Pastos pertenecientes al Resguardo de Túquerres, Nariño, Colombia. La unidad de estudio a la totalidad de médicos tradicionales, es decir 19 médicos, entre los cuales hay hierbateros, espirituales, yageceros y parteras.

Se realizó una minga de pensamiento, trabajo en equipo, esta técnica participativa reunió médicos espirituales, parteras, sobadores y hierbateros, quienes de manera espontánea explicaron la importancia de su ocupación, los elementos materiales, instrumentos, plantas, hierbas que utilizan para la prevención y tratamiento de las enfermedades más frecuentes de la comunidad, según su ocupación, igualmente representaron el ceremonial o ritual que emplean para el tratamiento de mencionadas enfermedades.

\section{Resultados y discusión}

\section{Estado actual de la medicina tradicional}

La medicina tradicional y ancestral se ocupa de aquellas prácticas y expresiones en todas sus especialidades, cuya finalidad es mantener las condiciones de salud de una comunidad, mediante el uso de medicinas tradicionales con base en derivados de plantas, animales o cualquier combinación entre ellos, en condiciones de calidad, seguridad, accesibilidad y responsabilidad.

Para muchas comunidades indígenas, la salud depende de las buenas relaciones que el hombre mantenga con la naturaleza y su comunidad. Según su cosmovisión existen lugares sagrados, habitados por espíritus de antepasados por los que se deben transitar con mucho respeto y sólo a determinadas horas, en caso contrario se corre el peligro de contraer enfermedades que se producen por la violación de espacios sagrados.

El concepto sobre salud gira en torno al bienestar de la persona cuando está en armonía consigo mismo, con Dios y la naturaleza, esto permite que la persona viva equilibrio con la madre tierra. La salud permite que la persona se aleje de todo aquello que le hace daño. Así, la comunidad percibe la enfermedad como un desequilibrio o desacato de las normas o leyes naturales, espirituales y morales, lo que da origen a una ruptura de la armonía del cuerpo, el alma y el espíritu. De una manera muy figurativa un médico tradicional pinta el concepto de enfermedad con la tristeza. 


\section{Importancia de la medicina tradicional}

La población de médicos tradicionales plantea que la medicina tradicional es importante porque ella busca restablecer y aplicar una Ley que Dios estableció para que los seres humanos se sintonicen con la Pacha Mama y vivan en armonía con ella. Además se trata de un desempeño que ofrece grandes beneficios para la salud de manera efectiva y económica; no causa daño, es natural, permite curar, revitalizar el cuerpo y el espíritu.

Los testimonios y evidencias recogidos de los médicos tradicionales permiten destacar la importancia de la medicina tradicional, ya que permite restablecer las leyes divinas y sintonizar la armonía entre los seres humanos y la Pacha Mama. La naturaleza ha sido muy generosa con el hombre y le ha brindado todos los bienes para se aproveche, son efectivos y económicos, no causan daños colaterales y además permiten sanar y revitalizar.

Se destaca la importancia que reviste la ocupación de cada uno de los médicos tradicionales en razón de que su ocupación y desempeño les permite atender cualquier necesidad de la comunidad, en estas condiciones se ven obligados a estar en permanente aprendizaje para casos de emergencias. Es de denotar que el objeto y fin del médico tradicional es querer, amar, servir y sanar a la comunidad.

\section{Síndromes sociales más frecuentes}

Cuando se aborda el tema de síndromes socioculturales, se hace referencia a un término de antropología médica que atañe a una enfermedad que afecta a una sociedad y cultura específica. Por lo general, no existe una alteración bioquímica, orgánica o funcional de los pacientes. La enfermedad no se encuentra presente en otros grupos sociales y culturales distintos al lugar en donde esta ha sido detectada, aunque si puede haber experiencias que tengan similitudes.

Al preguntar sobre las enfermedades más frecuentes en la comunidad de los Pastos Resguardo de Túquerres, estás las clasifican en dos grupos bien definidos: por una parte están aquellos síndromes socioculturales espirituales entre los que se encuentran, el espanto, el mal viento, el miado del cueche, el maleficio, el mal hecho y algunos síndromes que denominan "del alma" como la envidia, la soledad y la tristeza. Por otra parte, están aquellas enfermedades que denominan "del cuerpo", como la gastritis, úlceras, apendicitis, vesícula, cáncer y fracturas y luxaciones.

Los médicos tradicionales entrevistados aseguran que las enfermedades espirituales no las puede sanar la medicina occidental, estas sólo pueden ser tratadas mediante la sabiduría ancestral, incluso, muchas de las enfermedades del cuerpo pueden ser sanadas mediante la medicina tradicional. Con el objeto de dar claridad al concepto de 
cada uno de los síndromes socioculturales enunciados, se procede a definirlos según el criterio de los mismos médicos tradicionales entrevistados.

El espanto: También conocido como susto, según la tradición de la comunidad de los Pasto, es una enfermedad que padecen los animales y las personas, especialmente los niños, que consiste en la pérdida del alma, ya sea de forma parcial o total, esto es causada por una gran impresión o un miedo profundo. El espanto puede causar diarrea, vómito y falta de ganas, escalofrío, fiebre, pesadillas preferencia por lugares oscuros. Esta sintomatología no siempre es la misma en todas las personas. El espanto puede ser causado por espíritus, animales, por los muertos, hechiceros, entre otros.

El mal viento: También conocido como mal aire o simplemente aire. El mal aire es una enfermedad de posesión que consiste en que, un aire pesado, dañino y maléfico entra en el cuerpo de la persona dando origen a síntomas como el dolor de cabeza, reumas, mareos, malestar en general. Este síndrome solo puede ser tratado por el médico tradicional mediante un ritual destinado a sacar el mal del interior de la persona y llevarlo lo más lejano posible, o esconderlo, de tal manera que no pueda enfermar a otra persona.

El miado del Cueche: Refiere la tradición popular de los Pastos, que cuando sale el arco iris y se produce cierta llovizna, esta es muy peligrosa, porque es una especie de demonio o maña energía, que causa muchas enfermedades especialmente para niños, ancianos y mujeres en embarazo. Afirma la tradición que hay varios tipos de Cueche, el negro que siempre se pega en quebradas, el blanco en ríos pequeños, este último es el más peligroso y maligno, que al pegarse en la persona presentan síntomas de altas temperatura, llagas y granos en el cuerpo, que producen comezón y piquiña y que al quedarse dormidos soñaban con ataques de animales enfurecidos.

El maleficio: Conocido como hechizo o también mal de ojo, consiste en un ritual utilizado para dañar a otra persona física o espiritualmente con la intervención de mañas energías o el demonio y que causan muchas enfermedades. También es conocida esta enfermedad como brujería o mal hecho.

Enfermedades del alma: Según el criterio de los médicos tradicionales de la comunidad de los Pastos, existe una categoría de enfermedades espirituales que son las denominadas "enfermedades del alma", entre la que se encuentra la soledad, la envidia, la tristeza, el egoísmo, estas enfermedades se originan cuando hay descompensación interior en el ser humano, cuando se rompe la armonía del cuerpo, del alma, el espíritu y la naturaleza. Este tipo de síndrome causa daño tanto a la persona que lo padece como a las que lo rodean.

El tratamiento de enfermedades de tipo físico, corporal y espiritual en la comunidad de los Pastos, Resguardo Indígena de Túquerres está basado en elementos que 
da la naturaleza acompañados con un ceremonial o ritual característico. Los remedios se clasifican en dos grupos: por un lado están aquellos elementos naturales que ya han sido procesados en un laboratorio como en gastrizan y artrizan, para la gastritis $y$ artritis respectivamente. Por otro lado son aquellos elementos que se presentan en estado natural como el coguindo, el espigo y el chondú para el espanto acompañados con aguardiente y el repollo morado para la gastritis.

Merece destacarse el yagé como tratamiento para todo tipo de enfermedades corporales y espirituales, el yagé es una planta denominada científicamente Banisteriopsis caapi y es una especie botánica de la familia de las malpiquíaceas. Recibe varios nombres comunes según el área geográfica, tales como ayahusca, yagé, caapi, yaquque, mariti o pilde. Es una liana o enredadora de las selvas de Suramérica: Bolivia, Brasil, Colombia, Ecuador, Perú y Venezuela. Los médicos tradicionales cuidan esta planta con la que establecen una relación de simbiosis, en donde el beneficio del ser humano es la consciencia y el conocimiento de la selva, cuyo espíritu lo sanará. Con la misma elaboran la poción amazónica conocida como ayahuasca o purga.

El efecto de la purga medicinal con la ayahuasca es en general, descrito como mareo con abundantes y constantes vómitos, dependiendo del estado de quién se trate, puede desencadenar diarrea. La ayahuasca ha mostrado ser una terapia muy efectiva para múltiples enfermedades, en el campo de la psiquiatría, en el tratamiento de la depresión y la ansiedad, así como para problemas de la personalidad y la esquizofrenia. Problemas los cuales son los mismos que los médicos tradicionales nativos tratan, sólo que con otros nombres tales como susto o el miedo.

\section{Procedimiento diagnóstico y terapéutico}

Los elementos utilizados para el tratamiento de enfermedades en la comunidad objeto de estudio, se clasifican en dos grupos: aquellos elementos naturales que han sido procesados con antelación en un laboratorio y aquellos naturales sin procesar: los elementos naturales más usados son los siguientes:

Chilca: Cuando una comida cae mal y produce vómito y diarrea, cocinar 3 cogollos de chilca en un litro de agua durante cinco minutos tomar cuantas veces pueda.

Malva de olor: Se utiliza para los nervios. Machacar tres hojas de malva de olor y poner en medio vaso de agua fría y tomar una vez al día durante tres días.

Alfalfa: Sirve para contrarrestar la anemia. Sacar una copa de sumo de alfalfa y se toma con miel de abejas, una vez durante nueve días.

Mejorana: Se utiliza para los nervios. Se cogen tres cogollos de mejorana en infusión en un medio litro de agua y se toma tres veces al día con tres gotas de limón. 
Caléndula: Se utiliza para inflamaciones de frío. Se coloca tres cogollos con la flor en medio litro de agua en infusión, se toma tres veces al día durante tres días. Endulzar con panela.

Flor de tilo: La flor sirve para la bronquitis. Se cogen dos flores de tilo, se cocina durante cinco minutos en medio litro de agua y se toma tres veces al día durante nueve días.

Hierbabuena: Es un desparasitante, se saca una copa de sumo de hierbabuena y tomarse en ayunas.

Cedrón: Para el insomnio se coge dos cogollos de cedrón y cocinar en un medio litro de agua de panela durante cinco minutos, se toma todas las noches.

Toronjil: Se utiliza para los nervios, se coge tres cogollos y se cocina durante tres minutos en un litro de agua y se toma tres veces al día durante nueve días.

Llantén: Desinflama la gastritis, se cogen tres hojas de llantén y se cocina en un litro de agua durante tres minuto, se toma tres veces al día durante nueve días, endulzar con panela.

Romero: Se utiliza para cólicos menstruales, se cogen dos cogollos de romero y se coloca en un medio litro de agua en infusión. Tomar dos veces al día durante los días de menstruación. Endulzar con miel de abejas.

Orégano: Se utiliza para la tos. Coger tres cogollos de orégano y cocinar en un medio litro de agua durante cinco minutos y tomar tres veces al día con miel de abejas, durante nueve días.

Es necesario reconocer el valor de la naturaleza y la importancia de su conservación para poder seguir alimentándose de ella, si se destruye su diversidad se perderá una gran fuente de medicamentos que pueden ayudar a la humanidad. Según la sabiduría tradicional-ancestral de la comunidad de los Pastos, resguardo indígena de Túquerres, la ceremonia o ritual para curar las enfermedades es uno de los elementos naturales más utilizados para el tratamiento de enfermedades. El suministro de plantas medicinales por lo general va acompañado por un ceremonial para curar las enfermedades. Algunos de ellos son los siguientes:

El proceso de sanación o curación de una enfermedad en la medicina tradicional posee un ceremonial o ritual. En el caso del parto es necesario acomodar al bebé para que adquiera la posición de parto, para ello utilizan el aceite de almendras, esto permite una ubicación correcta, posteriormente se suministra remedios naturales para curar a mamá. En enfermedades espirituales, siempre se inicia con el proceso de sahumar 
es decir, purificación de la persona utilizando el sahumerio, luego la invocación a Dios, al sol, al agua y a todos los espíritus de los taitas mayores. El ritual considera la invocación a la Pacha Mama, para que brinde todo el poder de sus elementos en aras de la curación de los seres humanos.

A continuación se trascribe la invocación a la Pacha Mama, efectuada en una minga de pensamientos

El misterio es el más grande, el misterio de la vida, ya que nacemos de un vientre materno y un vientre de nuestra madre tierra, les pido a todos los compañeros que dejemos los daños que le hemos hecho a ella como es desraizar los montes, destruir los páramos, también evitar las quemas porque ahí construimos lo más valioso que es el agua, ya que sin agua no habría vida y del agua también venimos, ya que el agua desde el vientre materno nos engendran nuestros padres y la fuente del agua es la vida. Les pido a las compañeras parteras, a los médicos tradicionales hacer unas pequeñas campañas para reforestación de nuestros páramos y montes, así sería que le pediríamos perdón a nuestra madre naturaleza, ya que dicen por ahí, Dios perdona pero la naturaleza nunca, porque naturaleza es sabia y de ahí venimos las comunidades indígenas, también decimos que ahí se conservan nuestras plantas sagradas como tanto comestible y seres vivientes como los animales, por eso les pido a todas comunidades que sigamos trabajando en bien de toda la gente.

\section{Elementos utilizados para prevenir la enfermedad}

Uno de los aspectos más importantes de la medicina occidental y tradicional ancestral es la medicina preventiva, a continuación se consigna algunos testimonios de la medicina tradicional. Los médicos tradicionales consideran que las medidas de prevención de la enfermedad radican básicamente en una buena alimentación para el caso del cuerpo y para el caso del espíritu estar en paz con uno mismo, con los demás, con Dios y la madre naturaleza. Recomiendan estrategias como tomar mucha agua, utilizar las plantas, respiración profunda, caminar descalzo, meditar y orar.

Según el criterio de los médicos tradicionales entrevistados, la prevención de la enfermedad debe centrarse en el uso adecuado de todo lo que ofrece la naturaleza, la comida sana y básicamente estar en armonía con uno mismo, con los demás, con Dios y con la naturaleza, en ello radica la conservación de la salud.

\section{Cuidados antes, durante y después del parto}

Las parteras es un desempeño de la medicina tradicional, de vital importancia entre las comunidades indígenas, ellas tienen como función traer la vida, cuidarla y preservarla 
tanto de la madre como de los hijos. A continuación se consignan algunos testimonios sobre los cuidados antes, durante y después del parto. Las parteras de la comunidad de los Pastos, resguardo indígena de Túquerres, tienen muy claro que poseen una responsabilidad muy grande, pues son las designadas por la madre naturaleza para traer la vida al mundo. En estas condiciones, saben que deben hacer seguimiento de la mujer embarazada desde la concepción, cuando nace y el niño y luego del parto.

La partera es una proveedora de vida, sus destrezas y conocimientos le permiten atender con eficiencia un parto especialmente en aquellas comunidades que viven en lugares apartados. Su trato es de confianza y humanizado, basado en su experiencia, práctica, aprendizaje permanentemente, su saber es adquirido con la experiencia y trato con otras parteras. Ellas comparten información con las madres, la familia y la comunidad, además comparten alternativas de salud y responsabilidades, técnicas de crianza, cuidados preventivos, lactancia y planificación familiar.

La partera cuida y verifica la salud de las madres y sus bebés durante todo el ciclo maternal y asiste el parto. Ella puede extender sus cuidados a toda la vida de la mujer y es reconocida como proveedora primaria de servicios de salud maternal por los miembros de su comunidad o por el país donde se practica.

\section{Sugerencias para mejorar la atención de los médicos tradicionales}

Con el objeto de mejorar la atención del médico tradicional, la comunidad entrevistada plantea lo siguiente:

- Que nos den un papel en el que conste nuestro oficio.

- Que las autoridades que no nos persigan.

- Que la religión no nos persiga porque cree que somos hechiceros.

- Que deberían permitir trabajar juntos con la medicina occidental porque lo que ellos no saben, nosotros sí y al contrario.

- Debemos tener mucho cuidado, porque también hay personas descuidadas y no atienden con responsabilidad.

- La sugerencia es estar siempre prevenido, practicando en una o en otra persona para estar práctico en las cosas.

- Dentro de nuestra comunidad en las Instituciones Prestadoras de Servicios (IPS) no tienen en cuenta a los médicos tradicionales, sugerimos que nos integren a los médicos tradicionales para poder un paso adelante.

- Para mejorar la presentación del servició, es necesario crear un módulo de salud propio y poder ejercer libremente, función que nos han encomendado nuestros taitas y madre naturaleza.

- Qué nuestra medicina sea reconocida y valorada por todo tipo de autoridades y nos dejen de perseguir. 


\section{Sugerencias para preservar la medicina tradicional}

La comunidad plantea lo siguiente para preservar la medicina tradicional ancestral:

- Enviarle un mensaje al gobierno nacional, que nos deje cultivar y proteger nuestros montes y ambientes naturales porque ahí nuestra vida, nuestra salud y nuestra propia naturaleza.

- Decirle al gobierno nacional que ahí estamos y que luchamos para ser reconocidos internacionalmente y recordarle que los primeros que estuvimos aquí somos los originarios indígenas, los afrodescendientes, quienes hicimos la medicina.

- Debemos apoyarnos los unos a los otros.

- Para mantener la medicina tradicional, nosotros tenemos que educar a nuestros niños, nuestra comunidad, para que no se olvide esa sabiduría en el tiempo y espacio.

- La sugerencia es que haya recursos y capacitaciones por parte del Estado y se estructuren escuelas y universidades con nuestros saberes populares.

- Que nos capaciten más, hay mucha gente que he curado y tengo testimonios, pero cada día necesito saber más.

- La sugerencia que tengo para mantener esa medicina dentro de la comunidad es ser serio, sincero, correcto, no mentirle a la gente, porque hay médicos que son mentirosos, estafadores, de todo se ve.

\section{Percepciones de la comunidad de los pastos sobre la práctica de la medicina tradicional}

La comunidad posee una visión bastante clara sobre la medicina tradicional y básicamente la valora como un legado cultural-ancestral que los identifica. También plantea que cuando hay enfermedades graves o de intervención acuden a la IPS, incluso, cuando la enfermedad es muy grave el médico tradicional lo remite a la IPS, en caso contrario es atendido por el médico tradicional. Consideran que la medicina tradicional es una alternativa para la salud, que se trata de un bien o regalo de la naturaleza para tratar enfermedades del cuerpo y el espíritu a través de plantas cuyo poder de curación es ampliamente reconocido.

Con relación a los médicos tradicionales, opinan que poseen una sabiduría tradicional-ancestral aunque no pueden ejercer libremente por cuando no han sido certificados y son perseguidos por las autoridades y la iglesia, de igual manera los médicos occidentales no los aceptan. En cuanto a los conceptos de salud y enfermedad, estos se basan en la armonía del cuerpo con la naturaleza, cuando mencionada. Una vez más surge la inquietud de la comunidad por la falta de integración entre la medicina tradicional y la occidental, la comunidad manifiesta que el médico tradicional aún no es aceptado especialmente por los organismos estatales y la iglesia Católica y aún no han sido certificados, quedando este aspecto como una tarea a cumplir a mediano plazo. 
La medicina tradicional está adquiriendo, con el paso del tiempo el espacio que debe ocupar como una manifestación cultural de vida ancestral, pues ella abarca una amplia variedad de terapias y prácticas características de esta región. Aquí medicina tradicional se viene utilizando desde muchísimos años y sus practicantes han contribuido enormemente a la salud de la comunidad y la preservación de la cultura e identidad de la misma.

En 2002, la Organización Mundial de la Salud OMS, lanzó una estrategia sobre medicina tradicional para facilitar a los países la exploración de las posibilidades de esa medicina para mejorar la salud y el bienestar de la población, reduciendo a la vez al mínimo los riesgos de utilización de remedios de eficacia no demostrada o de una utilización inadecuada, la finalidad principal de la estrategia es alentar la realización de investigaciones.

En los países en desarrollo, donde más de un tercio de la población carece de medicamentos esenciales, la administración de remedios tradicionales y alternativos seguros y eficaces podría mejorar de forma importante el acceso a la atención a la salud.

\section{Conclusiones}

Con relación al estado actual en la práctica de la medicina tradicional del territorio de los Pastos, Resguardo Indígena de Túquerres, Nariño, Colombia, se logró establecer que en la región se desempeñan 19 médicos tradicionales en el desempeño de hierbateros, médicos espirituales, parteros y yageceros. Un solo médico tradicional puede desempeñarse en varios oficios.

Aún no se alcanza a percibir una brecha entre la medicina tradicional y la occidental, el estudio evidenció que el Estado, algunos sectores de la Iglesia y algunas Instituciones prestadoras de salud no aceptan esta práctica ancestral, llegando al punto de perseguir al médico tradicional acusándolo de hechicero, embustero y mentiroso. Se destaca la importancia de la medicina tradicional porque esta sabiduría ancestral permite restablecer las leyes divinas y naturales que rigen la relación entre Dios, la Pacha Mama y los seres humanos.

Existen dos categorías: los espirituales y los del cuerpo. Entre los más frecuentes del cuerpo está la gastritis, la úlcera, apendicitis, males de la vesícula, cáncer, fracturas y luxaciones. Entre los espirituales está el espanto, el mal viento, el miado del cueche, el maleficio o mal hecho y aquellas enfermedades que denominan "del alma", como la envidia, la soledad, la tristeza, el egoísmo, entre otros.

Se reconoce la gran cantidad de hierbas y su amplio espectro curativo entre los que se destacan la caléndula, la cola de caballo, la raíz china, la vira virá, el chontará, 
el yagé, el repollo morado, orégano, yerbabuena, manzanilla, alfalfa, romerillo, sábila, entre otros.

El acto curativo de una enfermedad va acompañado con un ritual o ceremonial, para el caso del parto es necesario preparar el bebé para acomodar para que adquiera la ubicación adecuada para su nacimiento para esto se utiliza aceite de almendras, para las enfermedades espirituales se purifica a la persona y el entorno con sahumerio, se invoca a Dios y a la naturaleza, el sol, el agua, y todos los espíritus de los mayores.

La prevención de la enfermedad se efectúa según el tipo de enfermedad, así por ejemplo para prevenir enfermedades del cuerpo se debe tener una buena alimentación, tomar mucha agua y consumir alimentos naturales. Para enfermedades del espíritu hay que estar en paz con uno mismo con Dios y la naturaleza, respiración profunda, caminar descalzo, meditar y orar. En cuanto al cuidado del parto, es necesario no apretarse el estómago antes del parto, tener una buena alimentación, caminar, hacer ejercicio, durante el parto, recibir el bebé con mucha limpieza, abrigarlo, limpiar las fosas nasales y después del parto hacer frotaciones en el estómago de la mamá con manzanilla.

\section{Recomendaciones}

- Socializar el presente estudio investigativo ante la comunidad y la IPS con el objeto de dar a conocer sus resultados e iniciar, planes de mejoramiento ante las debilidades encontradas.

- Iniciar con el proceso, ante las autoridades competentes la certificación de los médicos tradicionales.

- Para las autoridades del resguardo indígena de Túquerres, fortalecer los planes de capacitación para los médicos tradicionales en cuanto al uso de plantas medicinales, cultivo de las mismas en sus shagras y su procesamiento.

- Para los médicos tradicionales organizarse y presentar proyectos de capacitación ante las autoridades del resguardo para su respectiva financiación.

- Es necesario estructurar un modelo de salud propia que identifique a la región a través del cual se observe la integración y articulación de la medicina tradicional con la occidental; siendo este un pilar importante en la elaboración del Sistema Intercultural de Salud para Pueblos Indígenas (SISPI) a nivel nacional. 


\section{Lista de referencias}

Bernal, Ignacio, (y otros) -compiladores- (1991)-I. Memorias de los encuentros de médicos tradicionales indígenas del estado de Oaxaca. INI y Fondo de Naciones Unidas para el desarrollo infantil, UNICEF. Oaxaca.

Briones, G. (1998). Métodos de investigación, Trillas, Madrid.

Bown, Peter. J. (1998). Understanding medical antropology. Mayfield publishing. London.

Equipo Técnico Cabildo Indígena de Tuquerres (Coat., 2009). Plan de justicia y vida para el resguardo indígena de Túquerres (2009). Disponible en http://www.mininterior.gov.co/sites/default/files/plan_de_justicia_y_vida_para_e_ri_de_tuquerres_2009.pdf

Zubiría, R. (1999). La medicina en el descubrimiento de América. Fondo de Cultura Económica, México

Zuluaga, R, (1999). Las medicinas tradicionales. Instituto Colombiano de Cultura Hispánica, Bogotá, D. C. 